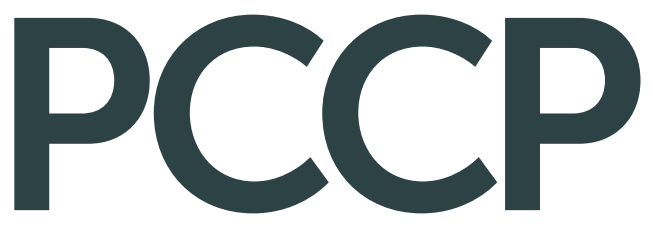

Physical Chemistry Chemical Physics rsc.li/pccp

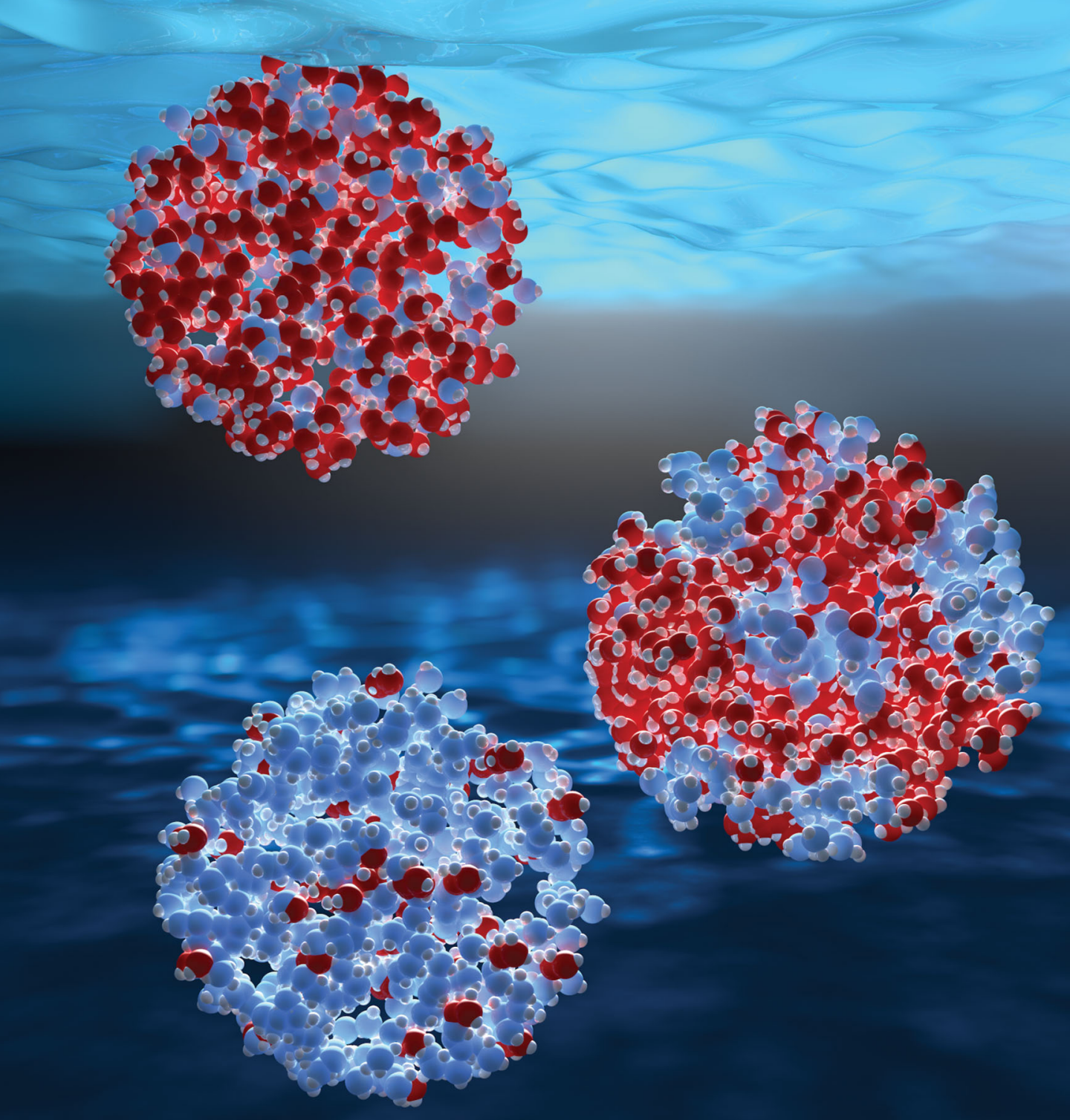

ISSN 1463-9076 
Check for updates

Cite this: Phys. Chem. Chem. Phys., 2019, 21, 26

Received 17th September 2018 Accepted 14th November 2018 DOI: 10.1039/c8cp05862h

rsc.li/pccp

\section{Apparent power-law behavior of water's isothermal compressibility and correlation length upon supercooling $\dagger$}

\author{
Alexander Späh, ${ }^{a}$ Harshad Pathak, (D) ${ }^{a}$ Kyung Hwan Kim, ${ }^{a}$ Fivos Perakis, (D) \\ Daniel Mariedahl, ${ }^{a}$ Katrin Amann-Winkel, (D) a Jonas A. Sellberg, iD b Jae Hyuk Lee, ${ }^{c}$ \\ Sangsoo Kim, ${ }^{c}$ Jaehyun Park, ${ }^{c}$ Ki Hyun Nam, ${ }^{c}$ Tetsuo Katayama ${ }^{d}$ and \\ Anders Nilsson (iD *a
}

\begin{abstract}
The isothermal compressibility and correlation length of supercooled water obtained from small-angle $X$-ray scattering (SAXS) were analyzed by fits based on an apparent power-law in the temperature range from $280 \mathrm{~K}$ down to the temperature of maximum compressibility at $229 \mathrm{~K}$. Although the increase in thermodynamic response functions is not towards a critical point, it is still possible to obtain an apparent power law all the way to the maximum values with best-fit exponents of $\gamma=0.40 \pm 0.01$ for the isothermal compressibility and $\nu=0.26 \pm 0.03$ for the correlation length. The ratio between these exponents is close to a value of $\approx 0.5$, as expected for a critical point, indicating the proximity of a potential second critical point. Comparison of $\gamma$ obtained from experiment with molecular dynamics simulations on the iAMOEBA water model shows that it would be located at pressures in the neighborhood of $1 \mathrm{kbar}$. The high value and sharpness of the compressibility maximum observed in the experiment are not reproduced by any of the existing classical water models, thus inviting further development of simulation models of water.
\end{abstract}

\section{Introduction}

Water behaves like a normal liquid at high temperatures but when cooled to lower temperatures, it shows anomalies in its thermodynamic properties such as density, heat capacity and compressibility which are enhanced upon decreasing the temperature further. ${ }^{1}$ At deeply supercooled temperatures, these properties seem to diverge. Speedy and Angell associated this divergence with an underlying thermodynamic singularity and described water's isothermal compressibility $\left(\kappa_{\mathrm{T}}\right)$ and other properties by a power law. ${ }^{2}$ Later, many more of water's anomalous quantities, including correlation length $(\xi)$, have been fitted by power laws with a vast majority yielding singularity temperatures $\left(T_{\mathrm{S}}\right)$ close to $228 \mathrm{~K} \cdot{ }^{3-6}$ However, the reliability of

\footnotetext{
${ }^{a}$ Department of Physics, AlbaNova University Center, Stockholm University, SE-10691 Stockholm, Sweden. E-mail: andersn@fysik.su.se

${ }^{b}$ Biomedical and X-Ray Physics, Department of Applied Physics, AlbaNova University Center, KTH Royal Institute of Technology, SE-10691 Stockholm, Sweden

${ }^{c}$ Pohang Accelerator Laboratory, Pohang, Gyeongbuk 37673, Republic of Korea

dJapan Synchrotron Radiation Research Institute, Kouto 1-1-1, Sayo,

Hyogo 679-5198, Japan

$\dagger$ Electronic supplementary information (ESI) available. See DOI: 10.1039/ c8cp05862h
}

such power-law fits and the existence of a hypothesized singularity had been questioned since homogeneous nucleation of ice precluded measurements close enough to the proposed $T_{\mathrm{S}}{ }^{7-11}$

To date, the liquid-liquid critical point (LLCP) scenario $^{12}$ is the most supported scenario ${ }^{13-16}$ among other models, ${ }^{17-19}$ which potentially explains water's anomalies. In this scenario, the hypothesized singularity is explained as a second critical point between two local and fluctuating configurations in the liquid - a high-density liquid (HDL) and a low-density liquid (LDL). According to the LLCP model, at low temperatures and elevated pressures, a LDL and a HDL exist as pure phases separated by a phase-coexistence line which terminates at the LLCP. Beyond the LLCP, at lower pressures, water is characterized by fluctuations between these HDL and LDL local structures. $^{1,20}$ The locus of maxima in $\xi$ of these fluctuations defines the Widom line in the pressure-temperature phase diagram, which emanates from the LLCP as an extension of the phase-coexistence line. Near the Widom line, also other lines of thermodynamic response function maxima exist and merge with the Widom line in close proximity to the critical point. ${ }^{21}$

In a study by Kim et al. ${ }^{13}$ such maxima in $\kappa_{\mathrm{T}}$ and $\xi$ have been found close to $229 \mathrm{~K}$, using small-angle X-ray scattering (SAXS) on evaporative cooled micron-sized water droplets ${ }^{22}$ in order to 
achieve temperatures all the way down to the proposed singularity temperature. This study has been criticized $^{23}$ but all issues could be addressed. ${ }^{24}$ Interestingly, the temperature of maxima in $\kappa_{\mathrm{T}}$ and $\xi$ was found to be very close to the $T_{\mathrm{S}}$ of $228 \mathrm{~K}$ that had been proposed earlier. This discrepancy between the finite, experimentally observed maxima and the divergence to infinity that would have been anticipated from the power-law extrapolation raises questions about the shape of the maxima in $\kappa_{\mathrm{T}}$ and $\xi$ as a function of temperature. In particular, investigation on the behavior of $\kappa_{\mathrm{T}}$ and $\xi$ around their maxima together with comparison to simulations may hint to the location of the LLCP in terms of pressure, if existent.

In the present study, we apply the commonly used power-law analysis to $\kappa_{\mathrm{T}}$ and $\xi$, but using the extended temperature range down to $229 \mathrm{~K}$ that has been reported recently. ${ }^{13}$ To stress that there is no critical point at ambient pressure and since power laws can only be fundamentally fitted close to a critical point, we will here instead use an apparent power law in the spirit of Speedy and Angell as a more empirical fit to investigate the steepness of the increase of the thermodynamic response functions. For $\kappa_{\mathrm{T}}$ and $\xi$, we use the notations ${ }^{25}$

$$
\kappa_{\mathrm{T}}=\kappa_{\mathrm{T}, 0} \varepsilon^{-\gamma} \text { and } \xi=\xi_{0} \varepsilon^{-\nu},
$$

where $\varepsilon=\left(T-T_{\mathrm{S}}\right) / T_{\mathrm{S}}$ is the reduced temperature, $T_{\mathrm{S}}$ is the singularity temperature and $\gamma$ and $\nu$ are the exponents. The results and the best-fit exponents are compared with previous studies on $\kappa_{\mathrm{T}}$ and $\xi$ at higher temperatures as well as MD simulations that together point to an LLCP located at a moderate positive pressure, in agreement with the estimation based on the value of $\kappa_{\mathrm{T}} \cdot{ }^{13}$

\section{Results and discussion}

\section{A. Apparent power-law analysis of experimental data}

Fig. 1 shows $\kappa_{\mathrm{T}}$ and $\xi$ of liquid $\mathrm{H}_{2} \mathrm{O}$ down to $\approx 250 \mathrm{~K}^{2,3,26}$ from thermodynamic measurements together with data obtained in the experiment by Kim et $a .^{13}$ with temperatures supercooled down to $\approx 227 \mathrm{~K}$. We note that the maximum in both $\kappa_{\mathrm{T}}$ and $\xi$ is rather sharp which makes it difficult to visualize in the broad plotted temperature range that is shown here (for more details see ref. 13). For temperatures below $250 \mathrm{~K}$ the power-law extrapolation given by Speedy and Angell, shown as the black dashed line in Fig. 1(a), deviates from the $\kappa_{\mathrm{T}}$ measurements reported by Kim et al. ${ }^{13}$ The temperature determination of the water droplets reported by Kim et al. is calculated using a ballistic evaporation model, see the ESI of ref. 13. Here we want to give a brief validation of the temperature estimate given by Kim et al. based on homogeneous nucleation rates, and a more detailed discussion on the temperature validation can be found in Section I of the ESI. $\dagger$ If we assume the temperature calibration by Kim et al. to yield too low temperature estimates and instead assume the temperature to match the power-law fit of Speedy and Angell, it would mean that the lowest temperature given by Kim et al. ${ }^{13}$ is close to $234 \mathrm{~K}$. However, with reported nucleation rates below $10^{10} \mathrm{~cm}^{-3} \mathrm{~s}^{-1}$ close to $234 \mathrm{~K},{ }^{27}$ we would expect a negligible fraction of the water droplets to crystallize before they are probed by the X-ray pulses. This would be in conflict with the very high crystallization probability that was observed for the lowest temperatures in the experiment by Kim et al. The factor $10^{3}$ higher homogeneous nucleation rates around $228 \mathrm{~K}^{28}$ are instead in full agreement with the observed nucleation probabilities at the lowest temperatures. We point out that the data reported by Kim et al. is derived only from liquid water droplets where all ice containing events have been sorted out.

The $\xi$ values measured by Huang et $a .^{3}$ are slightly larger than the current data, as shown in Fig. 1(b). Towards colder temperatures, the enhancement at low- $q$ rapidly grows and the choice of normal and anomalous components becomes increasingly independent, resulting in small error-bars of $\xi$. Using a slightly extended $q$-range to higher $q$-values, we found a slightly lower $\xi$ for the high-temperature data measured at PAL-XFEL than that reported previously. ${ }^{13}$ However, we note
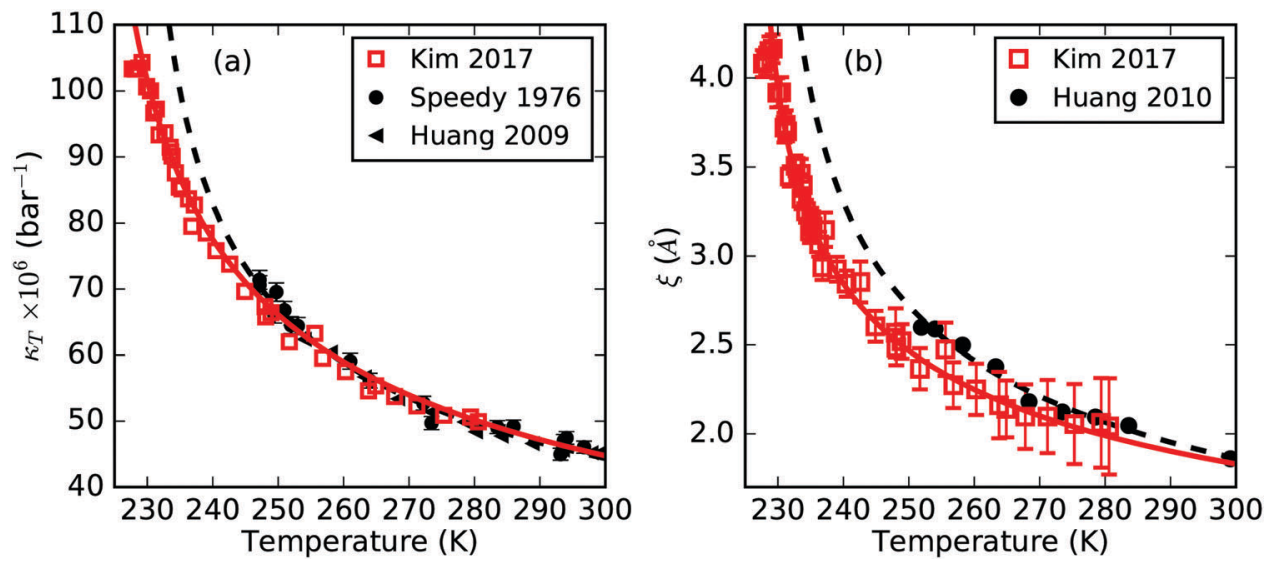

Fig. 1 Isothermal compressibility, $\kappa_{\mathrm{T}}$, (a) and correlation length, $\xi$, (b) obtained from SAXS on supercooled water droplets ${ }^{13}$ (squares). Error bars for $\kappa_{\mathrm{T}}$ are smaller than the symbol size. Literature data from Speedy and Angell ${ }^{2}$ and Huang et al. ${ }^{3,26}$ (full symbols) are shown for comparison. Apparent power-law fits with a singularity temperature $T_{\mathrm{S}}$ of $228 \mathrm{~K}$, reported by Speedy and Angell and Huang et al., are shown as dashed lines. Solid lines depict the least-squares power-law fits weighted by error bars for all shown data from the temperature of maximum $\kappa_{\mathrm{T}}$ or $\xi$ up to $300 \mathrm{~K}$. 
that the trend of increased $\xi$ towards lower temperatures is unaffected. For comparison, the black dashed line in Fig. 1(b) again indicates an apparent power-law fit with the suggested $T_{\mathrm{S}}$ of $228 \mathrm{~K},{ }^{3}$ deviating from the experimental data at low temperatures. Nevertheless, it is still possible to fit $\kappa_{\mathrm{T}}$ and $\xi$ by an apparent power-law including the extended temperature range, shown as solid lines.

We note that previous studies on $\kappa_{\mathrm{T}}$ of water ${ }^{4,29}$ first subtracted a normal component, which resembles the behavior of a normal liquid, before the remaining anomalous component was analyzed by an apparent power law. Following this procedure, it was found to yield unreasonably high exponents (see Section II of the ESI $\dagger$ ). Therefore, instead of segregating the normal and anomalous components, we decided to limit the temperature range for our apparent power-law analysis to the region where water's anomalous behavior is clearly apparent. For near-ambient pressures, this corresponds to temperatures ranging from the temperature of maximum $\kappa_{\mathrm{T}}$ or $\xi$ at $229 \mathrm{~K}$ all the way up to the highest measured temperature of $280 \mathrm{~K}$.

Even though the reported data were measured close to ambient pressure and thus far away from the hypothesized LLCP, it is possible to fit an apparent power law within measurement uncertainty up to $300 \mathrm{~K}$, shown as solid lines in Fig. 1. Interestingly we do not see a deceleration in the increase of $\kappa_{\mathrm{T}}$ or $\xi$ before they reach their maximum values at $229 \mathrm{~K}$, but instead find an accelerated increase with decreasing temperature according to an apparent power-law behavior all the way up to the maximum.

The best-fit exponents for $\kappa_{\mathrm{T}}$ and $\xi$ are $\gamma=0.40$ and $\nu=0.26$, respectively, and are summarized in Table 1 together with their singularity temperatures $T_{\mathrm{S}}$. The relatively small exponents obtained from the apparent power-law fits are in agreement with a moderate divergent behavior of the thermodynamic properties at ambient pressures and an LLCP at high positive pressure. At the LLCP the exponents are expected to become large and reach values of $\gamma \approx 1.24$ for $\kappa_{\mathrm{T}}$ and $\nu \approx 0.63$ for $\xi .^{30}$ The general relationship of $\nu / \gamma=1 /(2-\eta) \approx 1 / 2$ at a critical point is thus not exactly fulfilled in the current temperature and pressure regime, and instead the ratio $\nu / \gamma$ of the exponents is close to 0.65 . We also note that our best-fit results yield different $T_{\mathrm{S}}$ values for $\kappa_{\mathrm{T}}$ and $\xi$ as reported in Table 1.

It is also important to point out that the general interpretation of the singularity temperature $T_{\mathrm{S}}$ in the apparent powerlaw analysis implies a divergence of the thermodynamic response or correlation functions to infinity and is thus very different from the temperature $T_{\mathrm{W}}$ when approaching the Widom line, where the thermodynamic properties experience a finite maximum instead. In this context, $T_{\mathrm{S}}$ obtained from the apparent power-law fit is directly connected to the exponent.

Table 1 Best-fit parameters of $\kappa_{T}$ and $\xi$ according to the apparent power law given by eqn (1)

\begin{tabular}{llll}
\hline & $T_{\mathrm{S}}(\mathrm{K})$ & $\kappa_{\mathrm{T}, 0}\left(10^{-6} \mathrm{bar}^{-1}\right), \xi_{\mathrm{o}}(\AA)$ & $\gamma, \nu$ \\
\hline$\kappa_{\mathrm{T}}$ & $219.6 \pm 0.6$ & $29.9 \pm 0.4$ & $0.40 \pm 0.01$ \\
$\xi$ & $225.9 \pm 0.8$ & $1.36 \pm 0.08$ & $0.26 \pm 0.03$
\end{tabular}

Smaller exponents correspond to a slower increase with temperature followed by a sudden divergence upon approaching the singularity temperature $T_{\mathrm{S}}$. We would therefore expect a $T_{\mathrm{S}}$ that is closer to $T_{\mathrm{W}}$, as is the case for $\xi$, to yield a smaller exponent compared to the obtained exponent for $\kappa_{\mathrm{T}}$. In fact, only in the vicinity of the LLCP, the approximation of the thermodynamic properties by a power law is accurate and $T_{\mathrm{S}}$ and $T_{\mathrm{W}}$ coincide. This is further illustrated by the discrepancy of the experimental data compared to the apparent power-law fit when fixing $T_{\mathrm{S}}$ to the reported temperature of the maxima in $\kappa_{\mathrm{T}}$ or $\xi$, shown as dashed lines in Fig. 1 . The surprisingly close agreement between the experimentally observed temperature of maximum $\kappa_{\mathrm{T}}$ at $229 \mathrm{~K}$ and the proposed $T_{\mathrm{S}}$ of $228 \mathrm{~K}$ by Angell and coworkers ${ }^{2}$ is most likely caused by the slightly too high $\kappa_{\mathrm{T}}$ at their lowest temperatures and has already been noted by the authors themselves providing their proposed $T_{\mathrm{S}}$ of $228 \mathrm{~K}$ with rather large uncertainty.

\section{B. Apparent power-law analysis applied to molecular dynamics simulations}

It is of interest to evaluate the exponent of the apparent powerlaw fits of $\kappa_{\mathrm{T}}$ for various pressures and to compare the experimental data to the iAMOEBA water model. ${ }^{31}$ We note that none of the current MD models shows an accurate description of the experimental $\kappa_{\mathrm{T}}$ data. However, we chose this water model since it is known to equilibrate relatively fast even at temperatures below $230 \mathrm{~K}$. In addition, it was found to present the structure of supercooled water favorably in comparison to several other MD models. ${ }^{32}$

Fig. 2(a) shows the simulation data of $\kappa_{\mathrm{T}}$ at elevated pressures with increasing divergence close to the LLCP around 1700 bar. Apparent power-law fits are indicated as solid lines. The highest cut-off temperatures that are used for the fits relate approximately to the extent of the anomalous region at various pressures. Thus, closer to the critical point, we chose a narrower temperature range for the fit. The obtained singularity temperatures $\left(T_{\mathrm{S}}\right)$ and exponents $(\gamma)$ are shown in Fig. $2(\mathrm{~b})$. We find a linear behavior of $T_{\mathrm{S}}$ with pressure as a good approximation and find $\gamma$ approaching a reasonable value around $\gamma \approx 1.24$ (although with large uncertainty) close to the critical pressure of the iAMOEBA model, which adds further confidence to the chosen temperature ranges.

The apparent power-law exponent reaches the experimental value of $\gamma=0.40$ around $750 \mathrm{bar}$, which is approximately $1 \mathrm{kbar}$ away from the LLCP of the iAMOEBA water model. We therefore estimate the LLCP to be in the neighborhood of $1 \mathrm{kbar}$ in real water, assuming the same slope of $\gamma$ as a function of pressure. A similar pressure of 800 bar was found based on the variation of the $\kappa_{\mathrm{T}}$ maximum with pressure. ${ }^{13}$ Thereby, the location of the critical point in real water is estimated to exist at lower pressures than for the MD model. This indicates that the ratio between hydrogen-bond cooperativity and hydrogen-bond strength is much higher for real water. ${ }^{33}$

Fig. 3(a) depicts $\kappa_{\mathrm{T}}$ of several MD water models, $\mathrm{mW},{ }^{36}$ SPCE,${ }^{37}$ TIP4P $/ 2005,{ }^{38}$ E3B3 $^{39}$ and iAMOEBA, ${ }^{31}$ as well as the experimental findings. Although most of the models reproduce 

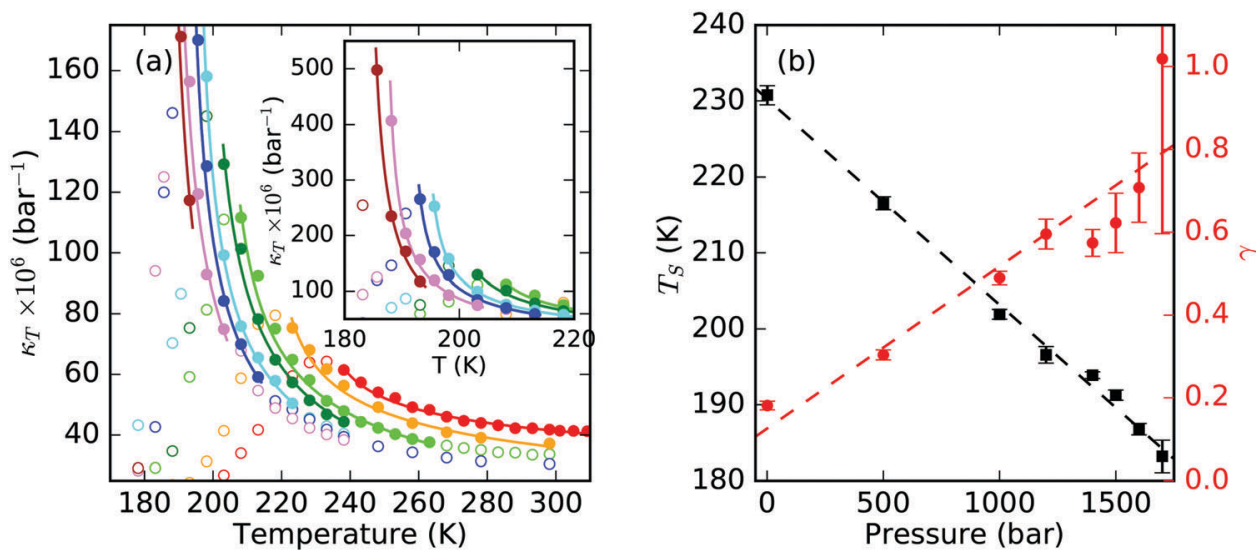

Fig. 2 (a) Isothermal compressibility, $\kappa_{T}$, of iAMOEBA water for pressures of 1, 500, 1000, 1200, 1400, 1500, 1600 and 1700 bar, ${ }^{13}$ shown in colors from red to brown, respectively. The error bars are not shown for simplification. The inset shows $\kappa_{\mathrm{T}}$ at a de-magnified scale for the lower part of the temperature range. Solid lines are apparent power-law fits to the $\kappa_{\mathrm{T}}$ data shown as solid points (hollow points are not used for fits). (b) Singularity temperatures (black squares) and exponents (red dots) obtained from apparent power-law fits for $\kappa_{\top}$ of the iAMOEBA water model. Dashed lines are a guide to the eye.
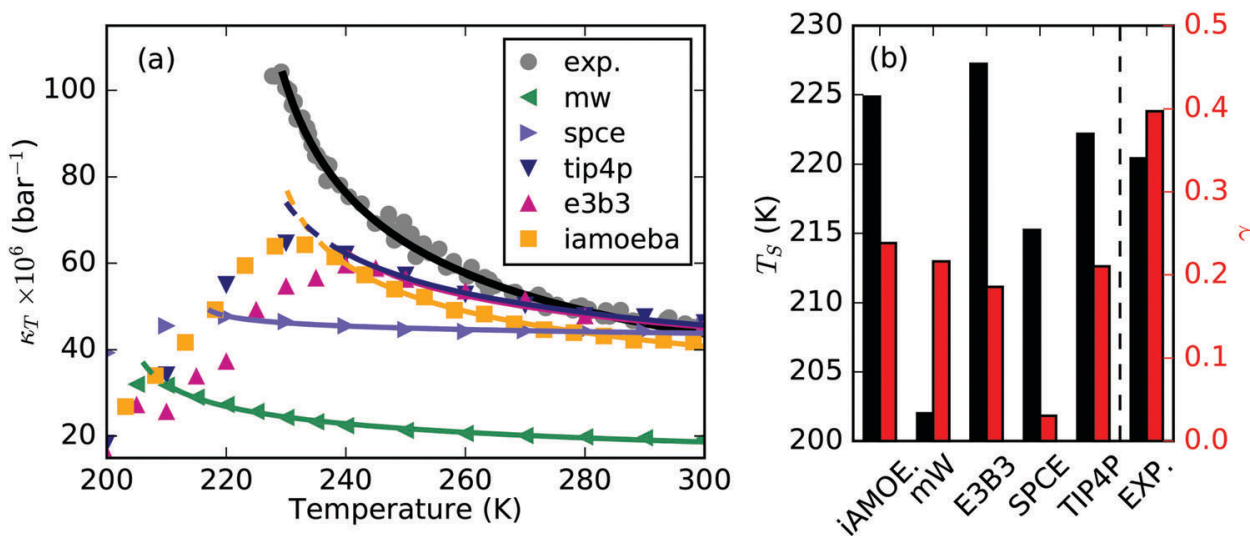

Fig. 3 (a) Isothermal compressibility, $\kappa_{\mathrm{T}}$, of various MD water models, ${ }^{32,34,35} \mathrm{~mW}$, SPCE, TIP4P/2005, E3B3 and iAMOEBA, compared to experimental values. Solid lines show the corresponding apparent power law fits in the temperature range from $300 \mathrm{~K}$ down to the temperature of maximum $\kappa_{\mathrm{T}}$. (b) Singularity temperatures and exponents obtained from the corresponding apparent power law fits given in (a).

$\kappa_{\mathrm{T}}$ above the melting temperature reasonably well, they deviate in their prediction upon supercooling. All of them underestimate $\kappa_{\mathrm{T}}$ compared to real water and exhibit only rather round maxima with respect to temperature as compared to experiments. The corresponding apparent power-law fits, shown as solid lines, yield very small apparent power-law exponents that are depicted in Fig. 3(b). In contrast, the experimental $\kappa_{\mathrm{T}}$ exhibits a strong and accelerated increase upon supercooling. To our knowledge the MB-pol water model ${ }^{40}$ seems to have the best description of $\kappa_{\mathrm{T}}$ but is left out in this discussion, since it is not clear whether the model shows a maximum at deeply supercooled temperatures where it is difficult to equilibrate the simulations. We also note that the WAIL water model $^{41}$ predicts an extremely high $\kappa_{\mathrm{T}}$ maximum that is beyond the experimental value. This is due to the close proximity of the LLCP, which lies at 500 bar for this model. Because of sparse data available for this model, it is also left out of the following discussion.

In addition to the underestimated $\kappa_{\mathrm{T}}$, all the depicted MD water models show a very broad maximum with respect to temperature, as compared to the sharp maximum that is observed experimentally. Although the experimental data exhibit an apparent power-law behavior all the way to the maximum, the simulation data start to round off and turn into a broad maximum already at temperatures 5-10 $\mathrm{K}$ before it reaches its maximum value. We can partially attribute the broad maximum in the simulations to the large distance in terms of pressure to the location of the LLCP in the models. Also, the choice of particular simulation conditions, i.e. system size, simulation time step, simulation and relaxation times, may partially contribute to a smoothening of the maxima in the simulations. In Fig. 3(a), the rounding-off is best seen for the iAMOEBA and TIP4P/2005 water models, where the dashed lines are continuations of the apparent power law fits.

The sharp maximum that is observed experimentally around the Widom line can be an indication of an increased clustering of hydrogen bonds due to increased intermolecular waterwater interaction energy, which has been interpreted in terms 
of a $\lambda$-like transition ${ }^{10}$ or by the concept of cooperative hydrogen bond connectivity. ${ }^{33}$ In particular, experimental data at lower temperatures would be necessary for quantitative analysis on the sharpness of the $\kappa_{\mathrm{T}}$ maximum. On the other hand, the broad maximum in the simulation data might be related to too small simulation boxes, which limit the growth of the fluctuating structural regions. Also, longer equilibration times might be necessary at these low temperatures to allow for the heterogeneities to develop. However, simulation data for more densely spaced temperature intervals close to the Widom line are necessary to study the shape around the maximum in detail.

\section{Conclusions}

We studied the anomalous increase in isothermal compressibility $\left(\kappa_{\mathrm{T}}\right)$ and correlation length $(\xi)$ of pure water upon supercooling from the data taken from Kim et $a l^{13}$ We made use of the previously inaccessible low temperature region below $250 \mathrm{~K}$, which allows for apparent power-law analysis without artificial subtraction of a normal component. The results show that the data can be fitted to an apparent power law within measurement uncertainty and for the temperature range from $300 \mathrm{~K}$ down to the temperature of maximum $\kappa_{\mathrm{T}}$. From the least-squares apparent power-law fits for $\xi$ and $\kappa_{\mathrm{T}}$, we obtain the ratio of their exponents, $\nu / \gamma=0.65$, which is relatively close to the ratio $\nu / \gamma \approx 0.51$ that would be expected exactly at a critical point. ${ }^{30}$ In general, the anomalous increase in $\kappa_{\mathrm{T}}$ observed in MD simulations is significantly smaller than that found experimentally. Comparison of $\gamma$ obtained from experiment and from MD simulations on the iAMOEBA water model indicates an LLCP located at pressures in the neighborhood of $1 \mathrm{kbar}$. The high value and sharpness of the $\kappa_{\mathrm{T}}$ maximum that is observed experimentally are not reproduced by any of the MD simulations, suggesting a slightly different behavior near the Widom line not predicted by the models. Both experiments at even lower temperatures and simulations with larger simulationbox size and much finer temperature resolution would be valuable to further investigate the sharpness of the $\kappa_{\mathrm{T}}$ maximum.

\section{Conflicts of interest}

There are no conflicts of interest to declare.

\section{Acknowledgements}

This work has been supported by the European Research Council (ERC) Advanced Grant under Project No. 667205 and the Swedish Research Council (VR) under Grant No. 2013-8823.

\section{References}

1 A. Nilsson and L. G. M. Pettersson, Nat. Commun., 2015, 6, 8998. 2 R. J. Speedy and C. A. Angell, J. Chem. Phys., 1976, 65, 851-858. 3 C. Huang, T. M. Weiss, D. Nordlund, K. T. Wikfeldt, L. G. M. Pettersson and A. Nilsson, J. Chem. Phys., 2010, 133, 134504.
4 H. Kanno and C. A. Angell, J. Chem. Phys., 1979, 70, 4008-4016.

5 C. A. Angell, W. J. Sichina and M. Oguni, J. Phys. Chem., 1982, 86, 998-1002.

6 A. Taschin, P. Bartolini, R. Eramo, R. Righini and R. Torre, Nat. Commun., 2013, 4, 2401.

7 D. H. Rasmussen and A. P. MacKenzie, J. Chem. Phys., 1973, 59, 5003-5013.

8 D. E. Hare and C. M. Sorensen, J. Chem. Phys., 1986, 84, 5085-5089.

9 P. Taborek, Phys. Rev. B, 1985, 32, 5902-5906.

10 C. A. Angell, J. Shuppert and J. C. Tucker, J. Phys. Chem., 1973, 77, 3092-3099.

11 B. J. Mason, Adv. Phys., 1958, 7, 221-234.

12 P. H. Poole, F. Sciortino, U. Essmann and H. E. Stanley, Nature, 1992, 360, 324-328.

13 K. H. Kim, A. Späh, H. Pathak, F. Perakis, D. Mariedahl, K. Amann-Winkel, J. A. Sellberg, J. H. Lee, S. Kim, J. Park, K. H. Nam, T. Katayama and A. Nilsson, Science, 2017, 358, 1589-1593.

14 P. Gallo, K. Amann-Winkel, C. A. Angell, M. A. Anisimov, F. Caupin, C. Chakravarty, E. Lascaris, T. Loerting, A. Z. Panagiotopoulos, J. Russo, J. A. Sellberg, H. E. Stanley, H. Tanaka, C. Vega, L. Xu and L. G. M. Pettersson, Chem. Rev., 2016, 116, 7463-7500.

15 F. Perakis, K. Amann-Winkel, F. Lehmkühler, M. Sprung, D. Mariedahl, J. A. Sellberg, H. Pathak, A. Späh, F. Cavalca, D. Schlesinger, A. Ricci, A. Jain, B. Massani, F. Aubree, C. J. Benmore, T. Loerting, G. Grübel, L. G. M. Pettersson and A. Nilsson, Proc. Natl. Acad. Sci. U. S. A., 2017, 114, 8193-8198.

16 S. Woutersen, B. Ensing, M. Hilbers, Z. Zhao and C. A. Angell, Science, 2018, 359, 1127-1131.

17 R. J. Speedy, J. Phys. Chem., 1982, 86, 982-991.

18 P. H. Poole, F. Sciortino, T. Grande, H. E. Stanley and C. A. Angell, Phys. Rev. Lett., 1994, 73, 1632-1635.

19 S. Sastry, P. G. Debenedetti, F. Sciortino and H. E. Stanley, Phys. Rev. E, 1996, 53, 6144-6154.

20 J. Russo and H. Tanaka, Nat. Commun., 2014, 5, 3556.

21 P. Gallo, D. Corradini and M. Rovere, Nat. Commun., 2014, 5,5806 .

22 J. A. Sellberg, C. Huang, T. A. McQueen, N. D. Loh, H. Laksmono, D. Schlesinger, R. G. Sierra, D. Nordlund, C. Y. Hampton, D. Starodub, D. P. DePonte, M. Beye, C. Chen, A. V. Martin, A. Barty, K. T. Wikfeldt, T. M. Weiss, C. Caronna, J. Feldkamp, L. B. Skinner, M. M. Seibert, M. Messerschmidt, G. J. Williams, S. Boutet, L. G. M. Pettersson, M. J. Bogan and A. Nilsson, Nature, 2014, 510, 381-384.

23 F. Caupin, V. Holten, C. Qiu, E. Guillerm, M. Wilke, M. Frenz, J. Teixeira and A. K. Soper, Science, 2018, 360, eaat1634.

24 K. H. Kim, A. Späh, H. Pathak, F. Perakis, D. Mariedahl, K. Amann-Winkel, J. A. Sellberg, J. H. Lee, S. Kim, J. Park, K. H. Nam, T. Katayama and A. Nilsson, Science, 2018, 360, eaat1729.

25 H. E. Stanley, Introduction to Phase Transitions and Critical Phenomena, Oxford University Press, New York, USA, 1971. 
26 C. Huang, K. T. Wikfeldt, T. Tokushima, D. Nordlund, Y. Harada, U. Bergmann, M. Niebuhr, T. M. Weiss, Y. Horikawa, M. Leetmaa, M. P. Ljungberg, O. Takahashi, A. Lenz, L. Ojamäe, A. P. Lyubartsev, S. Shin, L. G. M. Pettersson and A. Nilsson, Proc. Natl. Acad. Sci. U. S. A., 2009, 106, 15214-15218.

27 B. J. Murray, S. L. Broadley, T. W. Wilson, S. J. Bull, R. H. Wills, H. K. Christenson and E. J. Murray, Phys. Chem. Chem. Phys., 2010, 12, 10380-10387.

28 H. Laksmono, T. A. McQueen, J. A. Sellberg, N. D. Loh, C. Huang, D. Schlesinger, R. G. Sierra, C. Y. Hampton, D. Nordlund, M. Beye, A. V. Martin, A. Barty, M. M. Seibert, M. Messerschmidt, G. J. Williams, S. Boutet, K. AmannWinkel, T. Loerting, L. G. M. Pettersson, M. J. Bogan and A. Nilsson, J. Phys. Chem. Lett., 2015, 6, 2826-2832.

29 O. Conde, J. Teixeira and P. Papon, J. Chem. Phys., 1982, 76, 3747-3753.

30 D. Poland and D. Simmons-Duffin, Nat. Phys., 2016, 12, 535-539.

31 L.-P. Wang, T. Head-Gordon, J. W. Ponder, P. Ren, J. D. Chodera, P. K. Eastman, T. J. Martinez and V. S. Pande, J. Phys. Chem. B, 2013, 117, 9956-9972.
32 H. Pathak, J. C. Palmer, D. Schlesinger, K. T. Wikfeldt, J. A. Sellberg, L. G. M. Pettersson and A. Nilsson, J. Chem. Phys., 2016, 145, 134507.

33 K. Stokely, M. G. Mazza, H. E. Stanley and G. Franzese, Proc. Natl. Acad. Sci. U. S. A., 2010, 107, 1301-1306.

34 V. Holten, D. T. Limmer, V. Molinero and M. A. Anisimov, J. Chem. Phys., 2013, 138, 174501.

35 Y. Ni and J. L. Skinner, J. Chem. Phys., 2016, 144, 214501.

36 V. Molinero and E. B. Moore, J. Phys. Chem. B, 2009, 113, 4008-4016.

37 H. J. C. Berendsen, J. R. Grigera and T. P. Straatsma, J. Phys. Chem., 1987, 91, 6269-6271.

38 J. L. F. Abascal and C. Vega, J. Chem. Phys., 2005, 123, 234505.

39 C. J. Tainter, L. Shi and J. L. Skinner, J. Chem. Theory Comput., 2015, 11, 2268-2277.

40 S. K. Reddy, S. C. Straight, P. Bajaj, C. Huy Pham, M. Riera, D. R. Moberg, M. A. Morales, C. Knight, A. W. Götz and F. Paesani, J. Chem. Phys., 2016, 145, 194504.

41 Y. Li, J. Li and F. Wang, Proc. Natl. Acad. Sci. U. S. A., 2013, 110, 12209-12212. 